\title{
Determining Chemical Phases from Elemental Maps of Tablets
}

\author{
R. Anderhalt, T. Nylese
}

EDAX Inc., 91 McKee Drive, Mahwah, NJ, 07430

The word "phase" can be variably described to allow us to divide our sample into subareas that are distinct in terms of chemistry, crystallography, image gray level or color. As discussed here, we will limit the discussion to phases defined by chemical composition. Some of the first attempts to define chemical phases have been to use x-ray maps of individual elements to create an overlay whereby we select an element that is a unique identifier for each phase. Unfortunately, this requires some experience and knowledge by the operator and oftentimes the elements vary by only a slight degree between phases which can make the phase determination difficult at best. A variety of multivariate statistical techniques have been employed to create a more effective determination of phases [1]. Some of these techniques can be even be used during the original collection of the data. Even though pharmaceutical products are difficult to characterize because many active and inactive ingredients have very similar chemistry, it is possible to use EDS techniques to characterize these materials [2, 3]. On an even more fundamental level, many of these compounds are not stable under the electron beam or in a vacuum, and their x-ray yields tend to be minimal which makes even basic elemental mapping difficult and accurate phase determination extremely difficult.

A fractured surface of a multi-vitamin tablet was selected to illustrate phase maps. The sample was analyzed uncoated at a beam energy of $20 \mathrm{keV}$ using low-vacuum conditions (40 Pascal). X-ray mapping by EDS techniques of samples with a complex topographic surface is more difficult than mapping relatively smooth surfaces. Rough surface mapping is helped by the use of dual detectors which have an opposing view [4]. The combination of the two detectors will provide a better illumination of the sample surface than would be possible when using either of the two individual detectors (Fig. 1). A total of 13 phases were determined based on the chemical variation at each pixel and a different color is chosen for each phase (Fig. 2). All pixels that are assigned to a phase can be summed to show the phase chemistry and spectra for some of the most dominant phases are shown in figure 2 . The green phase is a magnesium oxide and the blue phase indicates an iron oxide. A calcium phosphate phase is shown in yellow while a potassium chloride phase is represented in a brownish red color. Other, more minor phases were high in $\mathrm{Cu}, \mathrm{Zn}, \mathrm{Mn}, \mathrm{Ca}, \mathrm{C}$, etc. A precise determination of the phase is made slightly more difficult by having performed the analysis in lowvacuum mode because of some scattering of the electron beam to other parts of the sample. Typically, the dominant elements are accurately determined. The relatively coarse nature of the multi-vitamin may provide a problem with determining a representative sample (Fig. 1) and it may be best to create a more representative sample by crushing a powder sample of the tablet.

\section{References}

[1] P.G. Kotula et al., Microsc. Microanal. (2003) 9 1-17.

[2] A.D. Vogt et al., Microsc. Microanal. 15 (Suppl 2) (2009) 380.

[3] J.P. Neilly et al., Microsc. Microanal. 15 (Suppl 2) (2009) 18.

[4] R. Anderhalt et al., Microsc. Microanal. 14 (Suppl 2) (2008) 1102. 

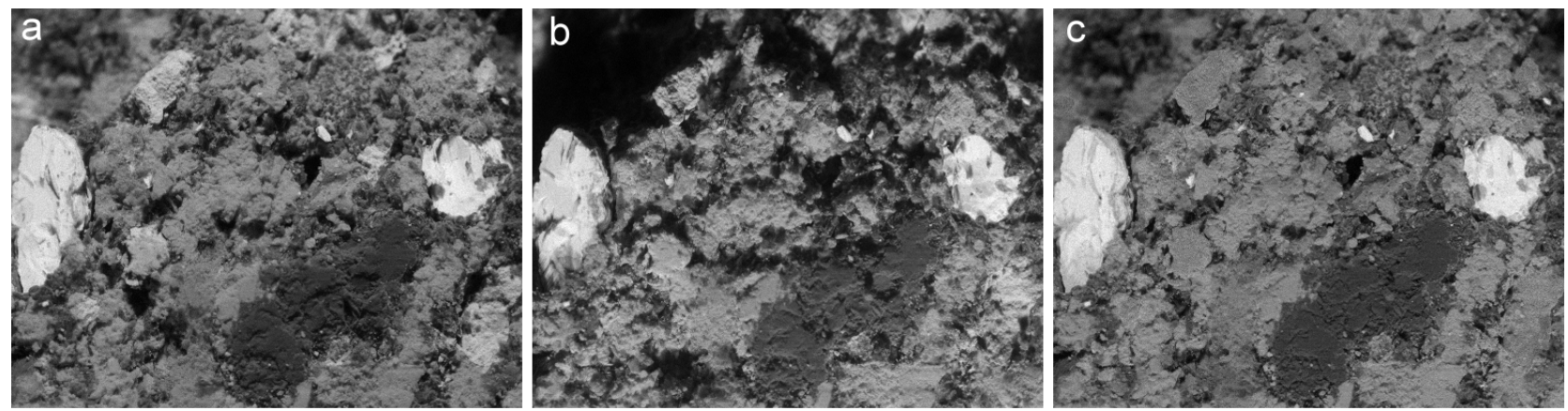

Fig. 1. Images derived from the total x-ray count rate detected at each pixel of the image (beam energy was $20 \mathrm{keV}$ ). In (a) the detector used was located above the image area and in (b) the detector was below the image area. The image in (c) is a combination of the two detectors and provides an even illumination of the sample surface. The image width in all images is $1.4 \mathrm{~mm}$.
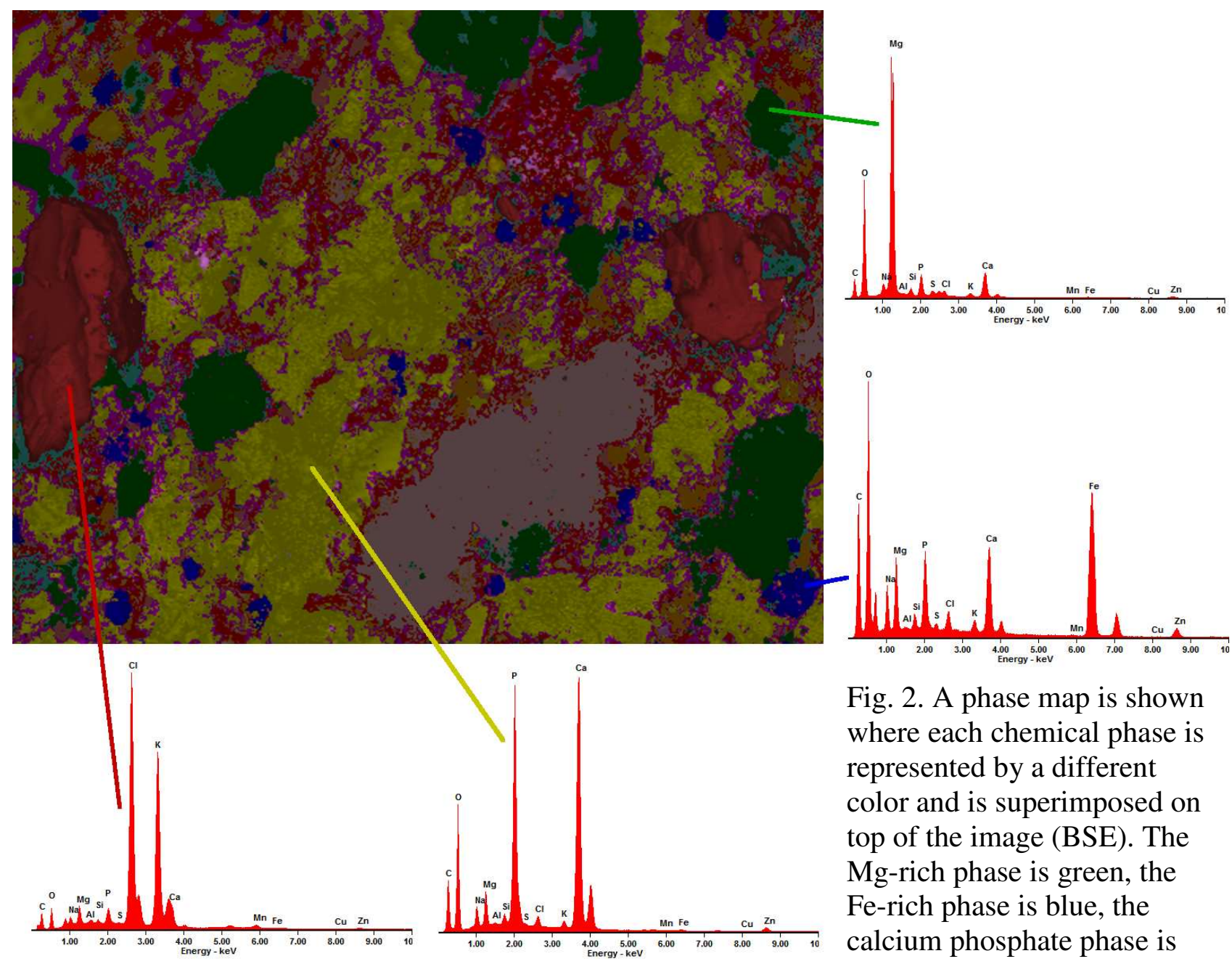

Fig. 2. A phase map is shown where each chemical phase is represented by a different color and is superimposed on top of the image (BSE). The $\mathrm{Mg}$-rich phase is green, the Fe-rich phase is blue, the calcium phosphate phase is yellow and a potassium chloride phase is a brownish red. The image width is 1.4 $\mathrm{mm}$. 